\title{
ON EXTENSIONS OF MODELS OF STRONG FRAGMENTS OF ARITHMETIC
}

\author{
ROMAN KOSSAK
}

(Communicated by Andreas R. Blass)

\begin{abstract}
Using a weak notion of recursive saturation (not always semiregularity) we prove that there are no finitely generated countable models of $B \Sigma_{n}+$ $\neg I \Sigma_{n}(n>0)$. We consider the problem of not almost semiregularity of models of $I \Sigma_{n}+\neg B \Sigma_{n+1}$. From a partial solution to this problem we deduce a generalization of the theorem of Smorynski and Stavi on cofinal extensions of recursively saturated models of arithmetic.
\end{abstract}

\section{INTRODUCTION}

By a strong fragment of arithmetic we mean every theory in the language of Peano Arithmetic extending $I \Sigma_{0}+B \Sigma_{1}+\exp$. The most well known strong fragments of arithmetic are $B \Sigma_{n+1}+\neg I \Sigma_{n+1}$ and $I \Sigma_{n}+\neg B \Sigma_{n+1}$, for all $n \geq$ 1. Classical examples of models of $B \Sigma_{n+1}+\neg I \Sigma_{n+1}$ and $I \Sigma_{n}+\neg B \Sigma_{n+1}$ are constructed as follows (cf. [PK]): If $a$ is an element of a model $M$ of $I \Sigma_{n+1}$, then

$$
\begin{gathered}
K^{n+1}(M, a)=\left\{X \in M: x \text { is } \Sigma_{n+1} \text {-definable in }(M, a)\right\} \\
I^{n+1}(M, a)=\left\{X \in M: \exists y \in K^{n+1}(M, a) x \leq y\right\} .
\end{gathered}
$$

For every $n>0$, if $K^{n+1}(M, a)$ is nonstandard then it is a model of $I \Sigma_{n}+$ $\neg B \Sigma_{n+1}$ and $I^{n+1}(M, a)$ is a model of $B \Sigma_{n+1}+\neg I \Sigma_{n+1}$. Also, since $K^{n+1}(M, a)$ is an $n+1$ elementary substructure of $M, K^{n+1}(M, a)$ is pointwise definable from $a$ and hence has at most countably many automorphisms. On the other hand, as was shown by Lessan in [L], if $M$ is countable, then $I^{n+1}(M, a)$ has continuum automorphisms. The main question that we consider in this paper is: to what extent are models of the form $K^{n+1}(M, a)$ and $I^{n+1}(M, a)$ representative for the classes of all countable models of $I \Sigma_{n}+\neg B \Sigma_{n+1}$ and

Received by the editors May 4, 1988 and, in revised form, December 12 1988. Presented to the American Mathematical Society, April 23, 1988, 843rd meeting, University of Maryland, Model Theory Session (Sponsored by David W. Kneker).

1980 Mathematics Subject Classification (1985 Revision). Primary 03C50, 03C52, $03 \mathrm{H} 15$.

Key words and phrases. fragments of arithmetic, recursive saturation, end extensions, cofinal extensions, automorphisms. 
$B \Sigma_{n+1}+\neg I \Sigma_{n+1}$, as far as the structural properties mentioned above are concerned? The answer is affirmative in the case of $B \Sigma_{n+1}+\neg I \Sigma_{n+1}$, all countable models of these theories have continuum automorphisms. Results toward the solution of the problem for models of $I \Sigma_{n}+\neg B \Sigma_{n+1}$ are much less satisfactory.

The existence of many automorphisms of models of the form $I^{n+1}(M, a)$ follows from their special property, which we have called in [K2] the $\omega$-property. A model $M$ has the $\omega$-property if in some end extension $N$ of $M$ there exists an element $a$ coding an increasing sequence of an infinite length and such that $M$ is the union of intervals of the form $\left[0,(a)_{n}\right]$, where $(a)_{n}$ is the $n$-th term of the sequence coded by $a$ and $n$ is a standard natural number (in [K2] we assumed that $N$ is an elementary extension of $M$; since the models we deal with here are not models of PA we have to drop this assumption). In particular, models with the $\omega$-property are not semiregular in some of their end extensions. We say that a model $M$ is not always semiregular if $M$ is not semiregular in some of its end extensions. All countable models with the $\omega$-property have continuum automorphisms. We will show that this is also true for all countable not always semiregular models and that all countable models of $B \Sigma_{n+1}+\neg I \Sigma_{n+1}$ are not always semiregular. We can ask whether the cofinality of a not always semiregular model must be the same in every one of its end extensions in which the model is not semiregular. Weak evidence that this might be true is given by the fact that there are models of $B \Sigma_{n+1}+\neg I \Sigma_{n+1}$ without the $\omega$-property (Section 6). One of the main open problems here is whether there are not always semiregular models of PA without the $\omega$-property.

Our main result about models of $I \Sigma_{n}+\neg B \Sigma_{n+1}$ says that there are countable models of these theories which do not have the $\omega$-property relative to a restricted class of their end extensions; in particular all countable recursively saturated models of $I \Sigma_{n}+\neg B \Sigma_{n+1}$ are such models. This contrasts with the fact that all countable recursively saturated models of PA have the $\omega$-property (cf. [K2]).

In [K2] we have argued that the $\omega$-property is a notion close to recursive saturation. Another evidence of this is given in $\S 4$, namely we prove that all countable models of PA with the $\omega$-property are isomorphic to all of their countable cofinal extensions with the same standard systems.

Results presented here follow the direction in the study of structural properties of models of arithmetic first taken by Lessan in his Ph.D thesis. This topic was popular about ten years ago and since then its popularity has certainly faded. Unfortunately Lessan's thesis, or its parts, have never been published, so many of its ideas and results deserving wider recognition are known only to a restricted group of specialists. This may be the reason why some of the interesting open problems (like those in [L, Chapter 5]) are still open. 


\section{Preliminaries}

We use the standard definitions of the collection and induction schemas for classes of arithmetical formulas, so we omit them here (in any doubts the reader may consult our main reference $[\mathrm{P}]$ and also $[\mathrm{PK}]$ or $[\mathrm{Cl}]$ ). For notational convenience we will use $B \Sigma_{n+1}+\neg I \Sigma_{n+1}$ to denote $I \Sigma_{0}+B \Sigma_{n+1}+\neg I \Sigma_{n+1}$.

Let $M$ be a model of $I \Sigma_{0}$. An element $a$ of $M$ is called small (cf. [L]) if elements of the form $2^{a^{n}}$, where $n \in \omega$, are bounded in $M$. If $a$ is small in $M$, then the structure $a_{M}=(a,+\times)$ is recursively saturated. We identify $a$ with the set $\{X \in M: x \leq a\}$ and consider + and $\times$ as ternary relations on $a$.

All models mentioned in the paper are models of $I \Sigma_{0}$.

2.1. Proposition. If $M$ is a model of $I \Sigma_{0}+\exp$ and $N$ is an end extension of $M$, then there are small elements in $N \backslash M$.

Proof. Indeed, for all $a$ in $M$ and any fixed $c, M \vDash \exists d 2^{a^{c}}<d$. Take any $e$ in $N \backslash M$. For all $a$ in $M, N \vDash \exists d<e \quad 2^{a^{c}}<d$, and by the $\Sigma_{0}$-overspill in $N$ this is also true for some $a$ in $N \backslash M$.

The standard system of a model $M$ of $I \Sigma_{0}, \operatorname{SSy}(M)$ is the collection of subsets of $\omega$, which are coded in $M$. The following proposition is a standard application of recursive saturation.

2.2. Proposition (Lessan, c.f. [L]). If $M$ and $N$ are models of $I \Sigma_{0}$, such that $M$ is a 0-elementary submodel of $N, \operatorname{SSy}(M)=\mathrm{SSy}(N)$, and $a_{M}$ and $a_{N}$ are countable and recursively saturated, then $a_{M}$ is isomorphic to $a_{N}$.

The assumption of $M$ being a 0 -elementary substructure of $N$ in the above proposition is superfluous when $M$ and $N$ are models of $I \Sigma_{0}+\exp$, because then if $M$ is a submodel of $N, M$ is also a 0-elementary submodel of $N$ (cf. [GD]).

Let us recall that a cut $I$ in a model $M$ of $I \Sigma_{0}$ is semiregular in $M$ if for every $a$ in $I$ and every function $f: a \rightarrow M$, which is coded in $M, f^{\prime \prime} a \cap I$ is bounded in $I$. The cofinality of $I$ in $M, \operatorname{cf}^{M}(I)$, is the set of those $a$ in $I$ for which there are no coded functions $f: a \rightarrow M$ such that $f^{\prime \prime} a \cap I$ is unbounded in $I$. So $I$ is semiregular in $M$ iff $\operatorname{cf}^{M}(I)=I$.

2.3. Proposition (Kirby, cf. [KP]). If I is a semiregular cut in a model $M$ of $I \Sigma_{0}$, then $I$ is a model of $I \Sigma_{1}$.

The following is a well known fine version of Gaifman's theorem.

2.4. Proposition (Gaifman, Dimitracopoulos [GD]). If $K$ is a cofinal extension of a model $M$ of $I \Sigma_{n}, n>1$, and $K$ is a model of $I \Sigma_{0}+\exp$, then $K$ is an $(n+1)$-elementary extension of $M$ and is a model of $I \Sigma_{n-1}$. 


\section{Models OF $B \Sigma_{n+1}+\neg I \Sigma_{n+1}$ ARE NOT ALWAYS SEMIREgUlaR}

Every model $M$ of PA has an elementary end extension $N$ such that $M$ is semiregular in $N$. For many models $M, M$ is semiregular in all of its end extensions. We call such models always semiregular. The existence of always semiregular models follows from

3.1. Theorem. Let $M$ be a model of $I \Sigma_{0}+\exp$ and suppose that $M \subseteq_{e} N$, where $N$ is a model of $I \Sigma_{0}$ and $M$ is not semiregular in $N$. Then for every finite sequence a of elements of $M(M, \mathbf{a})$ has a nontrivial automorphism.

Proof. By 2.1 we can select an $a$ in $N \backslash M$ such that $a_{N}$ is recursively saturated.

Claim. Let $\alpha, \beta \in a_{N}$ be such that there are no $\Sigma_{0}$-definable, $1-1$ functions from $\beta$ into $2^{\alpha^{n}}$ for all $n \in \omega$. Then for every finite sequence $\mathbf{b}$ of elements of $a_{N}$ there is an automorphism $f$ of $\left(a_{N}, \mathbf{b}\right)$ such that for some $e_{1}, e_{2}$ in $\beta_{N}, e_{1} \neq e_{2}, f\left(e_{1}\right)=e_{2}$ and $f$ restricted to $\alpha_{N}$ is the identity function.

Proof of the claim. Let $\operatorname{Tp}(x, \mathbf{b}, \alpha)$, the type of $x$ with parameters from $\alpha_{N} \cup \mathbf{b}$ in $a_{N}$, be the set

$$
\left\{\varphi(x, \mathbf{b}, \gamma): a_{N} \vDash \varphi(x, \mathbf{b}, \gamma) \& \gamma \in[\alpha]^{<\omega}\right\} .
$$

We have to show that there are $e_{1}, e_{2}$ in $\beta_{N}, e_{1} \neq e_{2}$, such that $\operatorname{Tp}\left(e_{1}, \mathbf{b}, \alpha\right)=$ $\operatorname{Tp}\left(e_{2}, \mathbf{b}, \alpha\right)$. Suppose not, then, by recursive saturation of $a_{N}$, there is an $r \in \omega$ such that, for all $x_{1}$ and $x_{2}$ in $\beta_{N}$, if $\operatorname{Tp}^{r}\left(x_{1}, \mathbf{b}, \alpha\right)=\operatorname{Tp}^{r}\left(x_{2}, \mathbf{b}, \alpha\right)$ then $x_{1}=x_{2}$, where

$$
\operatorname{Tp}^{r}(x, \mathbf{b}, \alpha)=\operatorname{Tp}(x, \mathbf{b}, \alpha) \cap\{\varphi(x, \mathbf{b}, \gamma): \varphi<r\} .
$$

So the function $f: \beta \rightarrow a_{N}$

$$
f(x)=\left\{[\varphi, \gamma]: \varphi(x, \mathbf{b}, \gamma) \in \operatorname{Tp}^{r}(x, \mathbf{b}, \alpha)\right\},
$$

where $[x, y]$ is a pairing function, is a $\Sigma_{0}$-definable in $N, 1-1$ function from $\beta$ into $r 2^{\alpha^{k}}$ for some standard $k$, contradiction.

Now, to prove the theorem, take an element $b$ in $N$ such that, for some $I \subseteq_{e} M, M=\sup \left\{(b)_{i}: i \in I\right\}$. Take $\alpha, \beta \in M$ such that $I<\alpha$ and $2^{\alpha^{n}}<\beta$ for all $n \in \omega$. Apply the claim for b such that $b_{0}=b$ (we can assume that $b$ is small enough in $N \backslash M$, so $b \in a_{N}$ ). Assumption of the claim is satisfied because the $\Sigma_{0}$-Pigeonhole Principle is a consequence of $I \Sigma_{0}+\exp$. Notice that if $f$ is as in the claim, for b chosen as above, then, since $f(b)=b$ and $f$ is identity on $I, f^{\prime \prime} M=M$, which finishes the proof.

If $M$ is countable and for every finite sequence a of elements of $M(M, a)$ has a nontrivial automorphism, then $M$ has continuum automorphisms (cf. $[\mathrm{Ku}])$. Hence we have the following corollary. 
3.2. Corollary. Countable models of $I \Sigma_{0}+\exp +B \Sigma_{1}+\neg I \Sigma_{1}$ are not semiregular in all of their end extensions, hence they have continuum automorphisms.

Proof. First of all, by [PW], all countable models of $I \Sigma_{0}+\exp +B \Sigma_{1}$ have end extensions to models of $I \Sigma_{0}$. The rest follows from Theorem 3.1 and Proposition 2.3.

For the generalization of the above corollary to the case of all theories $B \Sigma_{n+1}$ $+\neg I \Sigma_{n+1}$ we will need a strong result from [P]. The possibility of using it here was observed by L. Kirby. A cut $I$ in a model of $I \Sigma_{0}$ is 0 -extendible in $M$; it is said to be $(n+1)$-extendible if there is a $\Sigma_{0}$-elementary extension $K$ of $M$, such that $I$ is a cut of $K$, there is an $c$ in $K$ such that $I<c<M \backslash I$, and $I$ is $n$-extendible in $K$. Similarly, $I$ is $1 / 2$-extendible in $M$ if $I$ is semiregular in $M$ and $I$ is $(n+1+1 / 2)$-extendible if there is a $\Sigma_{0}$-elementary extension $K$ of $M$, such that $I$ is a cut of $K$, there is an $c$ in $K$ such that $I<c<M \backslash I$, and $I$ is $(n+1 / 2)$-extendible in $K$. It is known that a countable model $M$ is $I \Sigma_{0}$ is a model of $B \Sigma_{n+1}$ iff $M$ has an end extension in which it is an $n$-extendible cut; $M$ is a model of $I \Sigma_{n+1}$ if $M$ is $(n+1 / 2)$-extendible in some of its end extensions (cf. [P], in [P] $n$ is assumed to be $>0$, the result for $B \Sigma_{1}$ needs some additional assumptions like exp, see [PW]; a proof for $I \Sigma_{1}$ was given by Ratajczyk, unpublished).

3.3. Corollary. Countable models of $B \Sigma_{n+1}+\neg I \Sigma_{n+1}$ are not always semiregular, hence they have continuum automorphisms.

Proof. This follows directly from the facts mentioned.

\section{AN AMALgamation THEOREM}

For the proof of our next result we need a notion corresponding to the notion of a conservative extension for models of PA. Let $M$ be a model of $I \Sigma_{n+1}$ and let $N$ be an end extension of $M$; we will say that $N$ is an n-conservative extension of $M$ if $N$ is an $(n+1)$-elementary extension and for every finite sequence a of elements of $M$ and every $\Sigma_{n}$-formula $\varphi$ the set

$$
X_{\varphi, \mathbf{a}}=\{X \in M: N \vDash \varphi(x, \mathbf{a})\}
$$

is piecewise definable in $M$, i.e. for every $b$ in $M, X_{\varphi, \mathbf{a}} \cap b$ is coded in $M$. It can be easily shown that 1-conservative extensions must be end extensions.

If $M$ is a countable model of $I \Sigma_{n+1}$, then it is not difficult to construct a $\Sigma_{n}$-complete ultrafilter $U$ on $\Sigma_{n}$-definable subsets of $M$ (cf. [C2]) such that the ultrapower $M / U$ is $n$-conservative over $M$. Let us sketch this construction here. Let $M$ be a countable model of $I \Sigma_{n+1}$; we will construct a sequence of $\Sigma_{n}$-formulas with parameters from $M$. Let $\left[\varphi_{n}, a_{n}\right]$ be an enumeration of pairs of the form $\left[\Sigma_{n}\right.$-formula, element of $\left.M\right]$. Let $\Gamma(v)$ be the conjunction of the first $n$ formulas in the sequence and let $\left[\varphi_{n}, a_{n}\right]=[\varphi, a]$. We assume that $M$ satisfies $\mathrm{Q} v \Gamma(v)$, where $\mathrm{Q} v$ is the quantifier "there exists unboundedly many". Since $M$ is a model of $I \Sigma_{n+1}$ it satisfies also the induction schema for 
the closure under boolean operations and bounded quantification of the class of $\Sigma_{n+1}$ formulas (cf. [C1]), hence in $M$ we can define a coded subset $A$ of $M$ satisfying the following:

$$
\begin{gathered}
\{0 \in A \Leftrightarrow \mathrm{Q} v[\Gamma(v) \& \varphi(v, 0)]\} \& \forall x<a\{x+1 \in A \Leftrightarrow \mathrm{Qv}[\Gamma(v) \& \varphi(v, x+1)] \& \\
\forall j<x+1(j \in A \Rightarrow \operatorname{Qv}[\Gamma(v) \& \varphi(v, j) \& \varphi(v, x+1)])\} .
\end{gathered}
$$

Now we extend $\Gamma(v)$ by adding $\forall j \in A \varphi(v, j)$. Let $U$ be the collection of $\Sigma_{n}$-definable subsets of $M$ such that $X \in U$ iff for some $j \in A \quad X=\{x \in$ $M: \varphi(x, j)\}$, for some $A$ and $\varphi$ in the above construction. It is easy to check that $U$ has required properties. The same can be done for uncountable models except that the construction is not as easy (cf. [C2, the remark after Claim 1]).

4.1. Theorem. Let $N$ be a 1-conservative extension of a countable model $M$ of $I \Sigma_{n}, n>1$, and let $K$ be a cofinal extension of $M$, satisfying $B \Sigma_{2}$ (for $n>2$ this is satisfied automatically); then there is a model $L$ on $I \Sigma_{0}$ such that $N \subseteq L$ and $K \subseteq_{e} L$.

Proof. Let a be a finite sequence of elements of $N$; for every $\varphi$ in $\Sigma_{1}$ the set $X_{\varphi, \mathbf{a}}=\{x \in M: N \vDash \varphi(x, \mathbf{a})\}$ is piecewise coded in $M$. For every $\alpha \in M$ let $b_{\alpha, \varphi, \mathbf{a}}$ be a code for $X_{\varphi, \mathbf{a}} \cap \alpha$ and let $Y_{\varphi, \mathbf{a}}=\{x \in K: \exists \alpha \in M \quad K \vDash x \in$ $\left.b_{\alpha, \varphi, \mathrm{a}}\right\}$. Now consider the following theory $T$ in the language of arithmetic with constant symbols for all elements of $N \cup K$ :

$$
\begin{aligned}
T= & \operatorname{Th}(K, \mathbf{m})_{\mathbf{m} \in K}+\left\{\varphi(x, \mathbf{a}): x \in Y_{\varphi, \mathbf{a}}, \varphi \in \Sigma_{1}, \mathbf{a} \in N\right\} \\
& +\left\{\neg \varphi(x, \mathbf{a}): \neg x \in Y_{\varphi, \mathbf{a}}, \varphi \in \Sigma_{1}, \mathbf{a} \in N\right\} .
\end{aligned}
$$

Claim 1. $T$ is consistent.

Proof. It is enough to show that for $x \in Y_{\varphi, \mathbf{a}}$ and $y \in Y_{\psi, \mathbf{b}} \quad K \vDash \exists \mathbf{v}$, w $[\varphi(x, \mathbf{v}) \& \neg \psi(y, \mathbf{w})]$. Take $\alpha \in M$ such that $x, y<\alpha$. Then

$$
M \vDash \forall x, y<\alpha\left(x \in b_{\alpha, \varphi, \mathbf{a}} \& \neg y \in b_{\alpha, \psi, \mathbf{b}} \Rightarrow \exists \mathbf{v}, \mathbf{w}[\varphi(x, \mathbf{v}) \& \neg \psi(y, \mathbf{w})]\right) ;
$$

this is true because $N$ is a 2-elementary extension of $M$ and the above sentence is $\Sigma_{2}$ in $M$. Now, because $K$ is also a 2-elementary extension of $M$ and is a model of $B \Sigma_{2}$, the same sentence is true in $K$, proving the claim.

Let $L_{1}$ be a model of $T$ and let $L$ be the set of all elements of $L_{1}$ which are $\Sigma_{1}$-definable in $L_{1}$ using parameters from $K \cup N$ (we identify elements of $N$ with the constant symbols representing them). Since $L_{1}$ is a model of $I \Sigma_{1}$, $L$ is a model of $I \Sigma_{0}$.

Claim 2. $L$ is an end extension of $K$.

Proof. Suppose that, for some $x$ in $K$, some $\alpha$ in $M$ and a in $N$,

$$
L_{1} \vDash \varphi(d, x, \mathbf{a}) \& d<\alpha \& \exists ! v \varphi(v, x, \mathbf{a}),
$$

where $\varphi$ is a $\Sigma_{1}$-formula. We want to show that $d$ is in $K$. Let $\psi=\exists v<$ $w \varphi(v, u, \mathbf{t})$; then for some $\beta$ in $M$ and $\gamma$ in $M$ such that $x<\gamma$ we have

$$
M \vDash \forall x<\gamma\left([\alpha, x] \in Y_{\psi, \mathbf{a}} \Rightarrow \exists e<\beta[e, x] \in Y_{\varphi, \mathbf{a}}\right) .
$$


As in Claim 1, using codes $b_{\gamma, \psi, \mathrm{a}}$ and $b_{\gamma, \varphi, \mathrm{a}}$, we can show that

$$
K \vDash \exists e<b[e, x] \in Y_{\varphi, \mathbf{a}}
$$

hence $\varphi(e, x, \mathbf{a})$ is in $T$ for some $e$ in $K, e<b$. But then $L_{1} \vDash \varphi(e, x, \mathbf{a})$, so $e=d$, which finishes the proof of the claim and the proof of the theorem.

4.2. Corollary. Let $M$ be a model of $I \Sigma_{n}, n>1$, and suppose that $M$ has a cofinal subset of the order type $\omega$, which can be coded in a 1-conservative extension of $M$; then if $K$ is a cofinal extension of $M, K$ is a countable model of $B \Sigma_{2}$ and $\operatorname{SSy}(M)=\operatorname{SSy}(K)$, then $M$ is isomorphic to $K$.

Proof. Let $N$ be a 1-conservative extension of $M$ and let $L$ be as in the theorem for $M$ and $N$. Take $\alpha \in N \backslash M$ such that $M=\bigcup\left\{\left[0,(\alpha)_{n}\right]: n \in \omega\right\}$ and for an $a \in N \backslash M$ such that $\alpha \in a, a_{N}$ and $a_{L}$ are recursively saturated. Thus $a_{N}$ isomorphic to $a_{L}$ and if $f: a_{N} \rightarrow a_{L}$ is an isomorphism, then $f^{\prime \prime} M=$ $K$.

Similarly, one can prove that if a model of $I \Sigma_{n}, n>1, M$ is not always semiregular and for some 1-conservative extension $N \operatorname{cf}^{N}(M) \neq M$, then every countable model $K$ of $B \Sigma_{2}$ such that $M$ is cofinal in $K$ and $\operatorname{cf}^{N}(M)$ is properly included in the greatest common initial segment of $M$ and $K$, then $M$ is isomorphic to $K$.

4.3. Corollary. If $M$ is a countable model of PA and $M$ has a subset of the order type $\omega$ which can be coded in a 2-elementary end extension of $M$, then $M$ is isomorphic to all of its countable cofinal extensions with the same standard system.

Proof. Directly from Corollary 4.2.

In particular all countable models of PA with the $\omega$-property, as defined in [K2], are isomorphic to all of their countable cofinal extensions with the same standard system. The proof of Theorem 4.1 is a straightforward generalization of a corresponding amalgamation theorem for models of PA from [K1]; we believe that the assumptions of this result can be essentially weakened: for instance we expect that the answer to the following question is positive.

Question. Are all countable models of $I \Sigma_{0}$ with the $\omega$-property (as defined in this paper) isomorphic to all of their countable cofinal extensions with the same standard system?

\section{CofinAl EXTENSIONS OF MODELS OF $I \Sigma+\neg B \Sigma_{n+1}$}

Results from the previous section show that if a model $M$ has nonisomorphic cofinal extensions preserving some nonstandard initial segment $I$, then in all 1-conservative end extensions $N$ of $M$ we have $I<\mathrm{cf}^{N}(M)$. Every countable model of $I \Sigma_{n}+\neg B \Sigma_{n+1}$ has a cofinal extension to a model of $B \Sigma_{n+1}$. We will modify a proof of this well known conservation result to show that in many cases such a cofinal extension can preserve nonstandard initial segments. 
Let $M$ be a model of $I \Sigma_{n}+\neg B \Sigma_{n+1}$ for $n \geq 0$. By $\operatorname{Coll}_{n+1}(M)$ we will denote the set of those $a$ in $M$ for which

$$
M \vDash \forall x<a \exists y \varphi(x, y) \Rightarrow \exists b \forall x<a \exists y<b \varphi(x, y),
$$

for all $\Pi_{n}$ formulas with parameters from $M$. Observe that Coll $_{n+1}(M)$ contains all standard numbers and because $M$ is a model of $I \Sigma_{n}+\neg B \Sigma_{n+1}$, $\operatorname{Coll}_{n+1}(M)$ is always different from $M$. From the standard proof of the fact that $K^{n+1}(N, a)$ is a model of $I \Sigma_{n}+\neg \Sigma_{n+1}$, for $N \vDash I \Sigma_{n+1}$, it follows that $\operatorname{Coll}_{n+1}\left(K^{n+1}(N, a)\right)=\omega$. Also a simple "overspill" argument shows that if $M$ is a short recursively saturated model of $I \Sigma_{0}$ then, for any $n, \operatorname{Coll}_{n+1}(M)$ is a nonstandard initial segment of $M$.

5.1. Theorem. Let $M$ be a countable model of $I \Sigma_{n}+\neg B \Sigma_{n+1}$. There is a model $K$ of $B \Sigma_{n+1}$ such that $M$ is cofinal in $K$ and $\operatorname{Coll}_{n+1}(M)$ is included in the greatest common initial segment of $M$ and $K$.

Proof. We will follow the proof of [P, Theorem 11]. Let $\varphi$ be a $\Pi_{n}$ formula and let $a>\operatorname{Coll}_{n+1}(M)$ be such that

$$
M \vDash \forall x<a \exists y \varphi(x, y) \& \neg \exists t \forall x<a \exists y<t \varphi(x, y) .
$$

We will say that a coded subset $b$ of $a$ is $\varphi$-big if

$$
M \vDash \neg \exists t \forall x \in b \exists y<t \varphi(x, y) .
$$

It is shown in [P] that if $Z$ is an ultrafilter on the coded subsets of $a$ such that all members of $Z$ are $\varphi$-big then $N$, the ultrapower of $M$ with respect to $Z$, using coded maps from $a$ into $M$, is a model of $I \Sigma_{n}$ which is an $(n+1)$ elementary extension of $M$ and the instance of the collection schema for $\varphi$ and $a$ is satisfied in $N$. We will show that $Z$ can be chosen in such a way that $\operatorname{Coll}_{n+1}(M)$ is an initial segment of $N$. This will follow from the claim.

Claim. If $b$ is a $\varphi$-big coded subset of $a$ and for some $c$ in $\operatorname{Coll}_{n+1}(M)$ $b=\bigcup\left\{\beta_{a}: a<c\right\}$ is a coded partition of $b$, then some $\beta_{a}$ is $\varphi$-big.

Proof. Suppose that none of the $\beta_{a}$ 's is $\varphi$-big; then

$$
M \vDash \forall \alpha<c \exists \gamma \forall x \in \beta_{a} \exists y<\gamma \varphi(x, y) .
$$

Now, since $c$ is in $\operatorname{Coll}_{n+1}(M)$ all the $\gamma$ 's above can be bounded in $M$, contradiction.

The rest of the proof of the theorem is standard.

\section{MOdels OF $B \Sigma_{n+1}+\neg I \Sigma_{n+1}$ WITHOUT THE $\omega$-PROPERTY}

In this section we will sketch a proof of a result, which shows that there are not always semiregular models without the $\omega$-property. First let us note a general fact. 
6.1. Proposition. Among models of $I \Sigma_{0}+\exp$ which can be embedded as initial segments into a given countable model of $I \Sigma_{0}$, only countably many can have the $\omega$-property.

Proof. If $M \vDash I \Sigma_{0}+\exp , M \subseteq_{e} N \vDash I \Sigma_{0}$ and $a \in N$ witnesses the $\omega$-property, then, by 2.1 , we can assume that $a_{N}$ is recursively saturated. The theory of $a_{N}$ is then coded in the standard system of $M$, hence by the assumption, there are only countably many possibilities for the isomorphism type of $a_{N}$. But since the isomorphism type of $M$ is determined by the isomorphism type of $a_{N}$, the result follows.

6.2. Theorem. Every countable model of $I \Sigma_{n+1}$ has continuum many (nonelementarily equivalent) cuts which are models of $B \Sigma_{n+1}+\neg I \Sigma_{n+1}$ and do not have the $\omega$-property.

Proof. Let $\operatorname{Sat}_{n}(x, y)$ be a $\Sigma_{n}$-truth formula. Let $f_{n}(x, y)$ and $g_{n}(x)$ be the following functions:

$$
\begin{gathered}
f_{n}(x, y)=z \equiv\left[z=0 \& \forall t \neg \operatorname{Sat}_{n}(x,\langle y, t\rangle)\right] \text { or } \\
{\left[\operatorname{Sat}_{n}(x,\langle y, z\rangle) \& \forall z^{\prime}<z \neg \operatorname{Sat}_{n}\left(x,\left\langle y, z^{\prime}\right\rangle\right)\right]} \\
g_{n}(a)=c \equiv \exists x, y \leq a f_{n}(x, y)=c \& \forall z, y \leq a \exists t \leq c f_{n}(z, y)=t
\end{gathered}
$$

And finally let $G_{n}(x)$ be $g_{n}^{(x)}(x)$, which denotes $g_{n}(x)$ iterated $x$-times. Using $I \Sigma_{n+1}$ we can show that $G_{n}(x)$ is a total function, hence for every initial segment $I$ of $M, M_{n}(I)=\bigcup\left\{\left[0, G_{n}(i)\right]: i \in I\right\}$ is a proper initial segment of $M$. $B \Sigma_{n+1}$.

Claim 1. $M_{n}(I)$ is an $n$-elementary substructure of $M$ and is a model of

The first part of the claim follows from Tarski's test; proof of the second part uses the first part and is standard.

Claim 2. $I$ is $\Sigma_{n+1}$-definable in $M_{n}(I)$.

This follows from the fact that the formula defining the graph of $G_{n}(x)$ belongs to the class of formulas generated from $\Sigma_{n}$ by boolean operations and bounded quantification, and hence is absolute with respect to $n$-elementary end extensions. So we have: $i \in I$ iff $M_{n}(I) \vDash \exists x G_{n}(i)=x$.

$M$ has continuum many nonelementarily equivalent initial segments (for example it has an initial segment $N$ which is a model of PA and it is well known that among cuts of every countable model of PA there are models satisfying continuum different completions of PA). Thus the family $\left\{M_{n}(I): I \subseteq_{e} M\right\}$ contains continuum nonelementarily equivalent models. All models in this family are not semiregular in $M$, while on the other hand, by 6.1 , only countably many cuts of $M$ can have the $\omega$-property. 


\section{REFERENCES}

[C1] P. Clote, Partition relations in arithmetic, in Lecture Notes in Math. vol. 1130 Springer-Verlag, Heidelberg, 1985, pp. 32-68.

[C2] __ A note on the MacDowell-Specker theorem, Fundamenta Mathematicae 127 (1986), pp. 163-170.

[GD] H. Gaifman and C. Dimitracopoulos, Fragments of arithmetic and the MRDP theorem, Logic and Algorithmic, Monographie No. 30 de L'Enseignement Mathematique, pp. 187-206.

[KP] L. Kirby and J. Paris, Initial segments of models of Peano's axioms, in Lecture Notes in Math. vol. 619, Springer-Verlag, Heidelberg, 1979, pp. 211-226.

[K1] R. Kossak, A certain class of models of arithmetic, Journal of Symbolic Logic 48 (1983). pp. 311-319.

[K2] _ _ Models with the $\omega$-property, to appear in Journal of Symbolic Logic.

[Ku] D. Kueker, Back and forth arguments in infinitary logics, in Lecture Notes in Math. vol. 492, Springer-Verlag, Heidelberg, 1975, pp. 17-71.

[L] H. Lessan, Models of arithmetic, Ph.D. Thesis, Manchester 1978.

[P] J. Paris, Some conservation results for fragments of arithmetic, in Lecture Notes in Math. vol. 890, Springer-Verlag, Heidelberg, 1980, pp. 251-262.

[PK] J. Paris and L. Kirby, $\Sigma_{n}$-collection schemas in arithmetic, Logic Colloquium 77, North Holland, Amsterdam 1978.

[PW] J. Paris and A. Wilkie, A note on the end extension problem, (to appear).

Mathematics Department, Baruch College of CUNy, 17 Lexington avenue, NEW YORK, NEW YORK 10010 